\title{
Facial feminization procedures: the western approaches to the forehead
}

\author{
Han LT Hoang, MD \\ Institute of Cosmetic and Reconstructive Surgery, California, USA
}

Throughout the history, cross-dressing has been a practice that was well-accepted. However, gender dysphoria has only been recently approved by society. Facial feminization surgery (FFS) refers to a variety of surgical procedures aiming to transform the features of a male face to that of a female face. Several studies have illustrated that the upper third of the face plays the most importance role in determining gender. In this paper, the Western approaches to forehead feminization are discussed.

Level of Evidence: Level V

Keywords: facial feminization; forehead contouring; forehead reduction

\section{Introduction}

Throughout the history of Eastern and Western culture, crossdressing has been a well-accepted practice. However, gender dysphoria has only been recently approved by society. Gender reassignment surgery was first performed on Danish artist Einar Wegener, who unfortunately succumbed to infection following the surgeries. With the introduction of hormonal therapy and significant advancements in surgical techniques, transgender patients now have options for safe surgical interventions and better overall results.

Facial feminization surgery (FFS) refers to a variety of surgical procedures aimed at transforming the features of a male face to that of a female face. FFS was pioneered by Dr. Douglas Ousterhout from San Francisco, California. By studying male and female skulls, he identified differences in forehead contour and subsequently proposed treatment protocols $[1,2]$. Since the initial description in the 1980s, FFS has been popularized and has significantly evolved across all continents, particularly in Asia.

Due to testosterone, men are generally taller, more muscular, and have thicker bones. Facially, this translates to frontal bossing, lower eyebrows at the level of the orbital rim, prominent frontal nasal angles, larger noses, flatter cheeks, and wide, square jaws [3].

FFS may vary significantly from patient to patient. Several studies have illustrated that the upper third of the face plays an important role in determining gender $[4,5]$ This includes the shape of the hairline, the forehead, the eyebrows, the orbital roofs, and the root of the nose. In this paper, the Western approaches to forehead feminization are discussed.

\section{Forehead contour classification}

Dr. Ousterhout analyzed dry skulls of both sexes and classified forehead shape and contour into three distinct groups in his original description [1], with a fourth type subsequently 
added [6] (Fig. 1, 2):

- Type I foreheads have minimal or absent frontal sinuses, and therefore, contouring can be achieved with burring.

- Type II foreheads have protruding anterior walls of the frontal sinus, but normal frontal nasal angles. To improve the contour, augmentations of the area above the protrusion can be done with implants or bone cement.

- Type III foreheads have over-projected anterior walls of the frontal sinus. In this case, anterior wall osteotomy can be performed to set back and reshape the forehead.

- Type IV foreheads are small and under projected. Forehead contours can be improved with augmentation of the entire forehead with implants or bone cement.

In types I and III, some surgeons obtain pre-operative computed tomography (CT) scans to confirm the forehead types

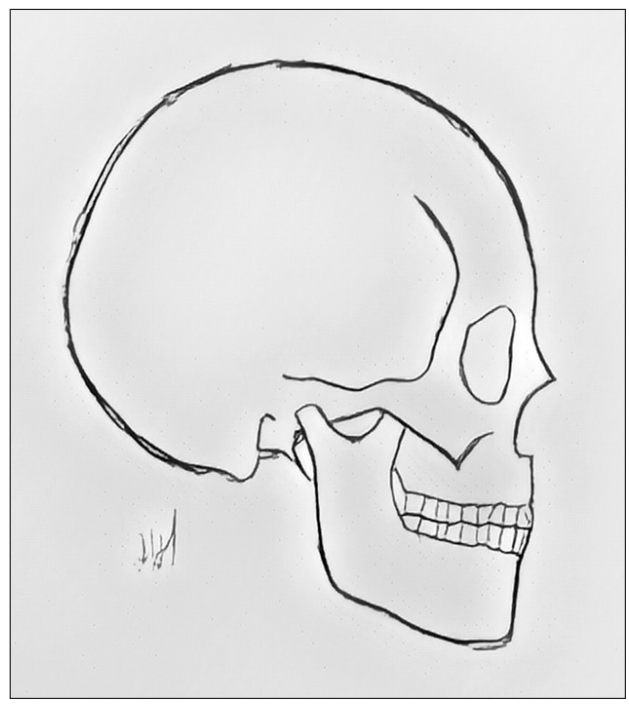

Fig. 1. Female skull. and plan for facial surgery $[7,8]$. Spiegel reported safe sinus intervention without pre-operative CT scan [4], though the author also recommended pre-operative imaging, either by cephalogram or CT scan, for surgical planning and pre-operative counseling.

\section{Forehead contouring}

The two common surgical approaches for forehead procedures are [4,7-10]:

1. Hairline approach, if hairline correction is required

2. Coronal approach, if forehead height is acceptable

Incisions should be made tangential to the hair follicles $[7,9,10]$, which would allow hair growth through the resulting scar. Hairline incisions can follow the temporal hairline, and then run in a posterior direction into the hair-bearing area and down towards the ears.

Local anesthetics with epinephrine should be injected along the planned incisions and along the plane of dissection to minimize bleeding. The author recommends dilution of $0.25 \%$ Marcaine with epinephrine, and a $50 \%$ saline mix, so that approximately $100 \mathrm{cc}$ can be safely injected into the scalp. In patients who require significant reduction with possible sinus intervention, pericranial flap should be considered as it can be raised quickly with flap elevation. In this case, scalp flaps should be raised in the subgaleal between temporal crests, and laterally connect to the superficial layer of the deep temporal fascia to avoid injury to the frontal branches of the facial nerve. At $1 \mathrm{~cm}$ above the orbital rims, the pericranial flap can be raised. Following elevation of the pericranial flap, the dissection plane is converted to a sub-periosteal plane. In severe bossing cases, it is more crucial to attain wide exposure of the entire frontal area down to the glabella, including the frontal processes of the zy-
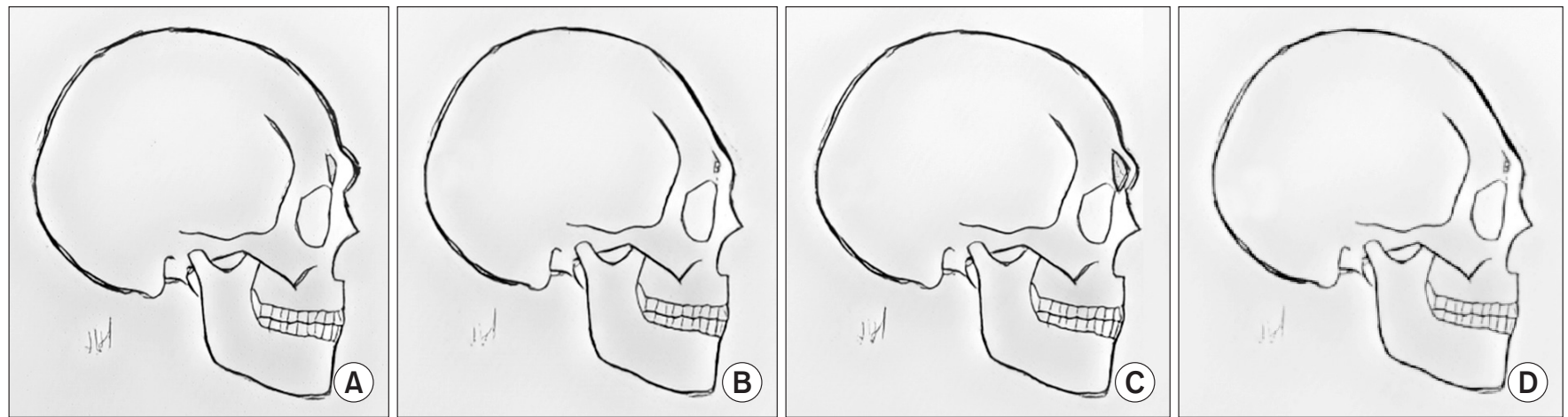

Fig. 2. Forehead shape classifica on (A) Type 1, (B) Type 2, (C) Type 3, (D) Type 4. 
gomas and the superior orbital rims, dissecting into the roof of each orbit. The supra-orbital nerves may need to be dissected or osteotomized out of their foramina in order to expose the entire superior orbital rims $[7,9]$.

Reduction of the bossing, including the frontal processes of the zygomas and the lateral superior orbital rims, can be burred to smooth away any bony infringement and to expand the orbital perimeter. Ousterhoust, Altman, Capitan, and Komuro reported the use of either pineapple or methylmethacrylate burr [1,7-9].

In type III foreheads, the size of each sinus and frontal nasal sinus duct can be assessed if CT scans are obtained. In most cases, the frontal nasal angle is acute [7-9], and therefore osteotomy planning should include the inferior aspect of the anterior table of the frontal sinus at the glabella, and immediately above the radix.

The anterior table cuts are marked out on the bone. These cuts can be made safely with a fissure burr or sagittal saw facing towards the sinus at all times in order to ensure correct positioning $[4,7,8]$. The author uses fissure burr, while Altman reported use of both, and Komuro used a sagittal saw. Once blue sinus mucosa is encountered, a fine osteotome (used by Altman and the author), sagittal saw (used by Komuro), or oscillating saw (used by Spiegel), can be used to perform the final osteotomy to separate the anterior table from the mucosal lining $[4,7,8]$. Mucosa can then be dissected off of the anterior table, exposing the septum. The septum can be reduced safely with zero-tominimal mucosal tearing. The anterior table bone flap can be further reduced in thickness and diameter and set back (Fig. 3).

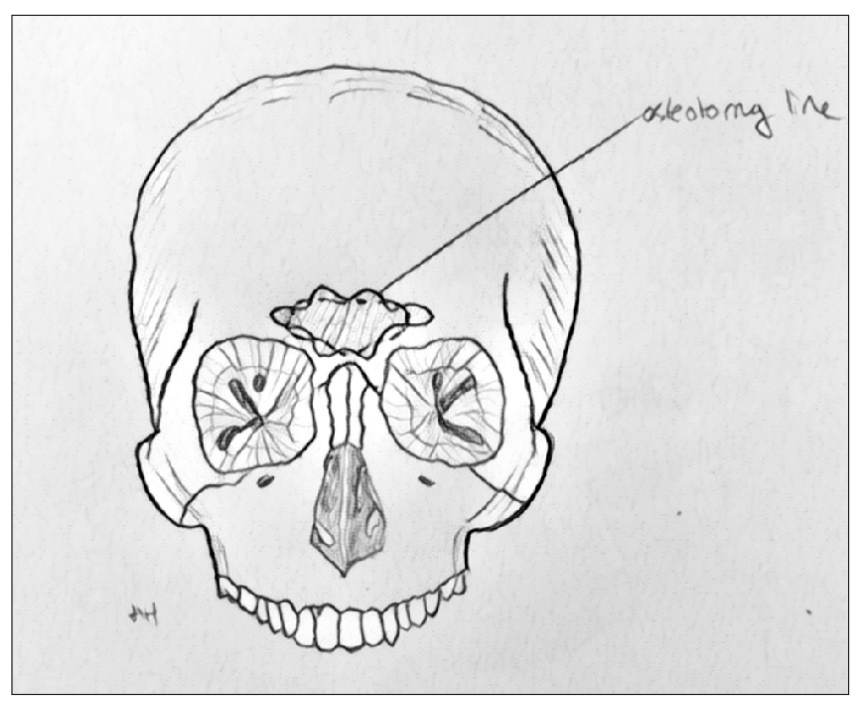

Fig. 3. Type 3 treatment with Osteoplas c flap.
Komuro described segmental reduction with the anterior wall divided into pieces.

The frontal sinus cavity and nasal frontal duct should be inspected carefully for any major mucosal injuries. If major injury occurs, obliteration versus post-operative endoscopic follow up should be considered. If minor tears occur, a pericranial flap can be used to separate the bone flap from the mucosa.

Next, the contoured anterior table can then be set back and secured with any low-profile titanium plate. The author recommends $1.3 \mathrm{~mm}$ titanium mini-plates with three points of fixation. The surrounding bone can also contoured, but a precise fit is not required, as steps or deficiencies cannot be appreciated on palpation via the scalp.

The outer third of the orbital rim can be burred down to increase the dimensions of the anterior orbital rim [7].

Spiegel reported his experience with the "islands" bone flap technique, described by a Thai surgeon, in which the anterior table is thinned down and manually broken into "islands" of bone without tearing the mucosa. In his experience, all of his non-union complications in the series were from this technique, and he has since abandoned this technique. Spiegel also recommended preserving the bone dust from the burring to create a bone paste in order to smooth over any gaps created by the osteotomy [4].

\section{Brow lift}

Brow lifting is also an important key step of forehead procedures. The female brow is more arched and cephalad compared to the male brow. It should sit above the supra-orbital ridge with highest peak at the lateral third. Elevating the brow, in conjunction with orbital rim contouring, are very effective in feminizing the upper face. Upper lid blepharoplasty may also complement this procedure [7].

A variety of brow lifting techniques have been described. One popular technique described by Altman is the dermal suturing technique [7]. In this technique, two bone bridges are drilled into the cranial vault a few centimeters superior to each brow, and a size 0 Ethibond suture (Ethicon Inc., Somerville, NJ, USA) is secured to the dermis at a point one-third of the distance from the outer aspect of the brow [7]. The dermal anchor is done using a large venous cannula introduced through the skin of the brow to the underside of the coronal flap. The suture can then pass through the cannula needle. The cannula is then withdrawn to the dermis and reinserted at the same time, picking up the dermis of the eyebrow. The suture can then be se- 
cured to the drilled bone bridge with appropriate tension. The brow elevation is prone to descending up to a few months postoperation. With this technique, Altman noted that there may be some initial dimpling of the eyebrow at the site of insertion, but this effect is temporary [7].

Brow lifts by skin excision with periosteal releases of scalp flaps can also be done, especially if hairline advancement is needed $[10,11]$. Flowers recommended a $4: 1$ skin excision without frontalis resection [10]. Nahai reported a variety of access incisions, and recommended pretrichial incision for patients with long foreheads [11].

\section{Hairline advancement}

If hairline advancement is required, it is important to assess scalp laxity to determine how far the scalp can be advanced [7]. On occasion, patients may require more than one operative procedure to attain the desired amount of advancement [7].

Although a pretrichial "hairline" incision prevents retrohairline displacement, it results in a brow-lifting effect, caused by shortening the forehead only and not true hairline lowering. However, when a pretrichial incision is used in combination with a posterior scalp advancement flap, true hairline lowering is possible.

Altman, Ramirez, and Marten all advocate "trichial" incision along the frontal scalp instead of true pretrichial incision. Frontal scalp incision should be made within the fine hair of the anterior scalp approximately 4-5 $\mathrm{mm}$ behind the true hairline beveling toward the scalp $[7,12,13]$. Then, the incision can be transitioned into the scalp in the temporal areas and turned inferiorly toward the root of the ears, where an incision is made parallel to the hair shafts. The scalp can be extensively undermined in the subgaleal plane back to the occiput, and if necessary, several parallel releasing incisions through the galea every $1.5-2.0 \mathrm{~cm}$ can assist scalp advancement and tension free closure $[7,12,13]$. Galeotomies are made through the galea just enough to expose underlying scalp fat and to preserve the blood supply to the scalp $[7,12,13]$. Each incision in the galea results in 1-2 mm of scalp flap expansion [13]. At this point, the amount of forehead skin to be excised can be determined prior to excision and closure. Marten described that maximal scalp advancement can be achieved with the division of the occipital vessels and nerve. He also employed a galea-anchoring technique for both the scalp advancement flap and the forehead flap using a mattress suture anchoring into monocortical bone tunnels [13]. Ramirez anchored the scalp flap using two Endotine devices in the paramedian position [12].

If extensive dissection is required, closed suction drains can be considered. They should be tunneled under a pericranial bridge to prevent migration. It is essential that the scalp is not sutured under tension. Altman described scalp closure without galea suturing, whereby monofilament sutures can be used for non-hair bearing skin areas, and staples can be used for hairbearing areas. The author recommends a two-layer closure including galea and skin suturing. A head bandage is placed for 24-48 hours, and if used, the vacuum drains should be removed at $24 \mathrm{~h}$. Post-operative sinus precautions should be given if indicated.

\section{Complications}

These procedures have relatively low major complication rate ranging from 0.0 to $1.8 \%[1,4,8,9]$. Capitán et al. [8] reported one case of cerebrospinal fluid fistula, which resolved with conservative management. Spiegel [4] reported three cases of non-union of the frontal sinus using the "islands" technique. There has been no report of total loss of the anterior table due to bone resorption or infection. The main negatives following surgery are forehead and scalp hypoesthesia that can last for months, and hair loss along the incision. With meticulous surgical techniques, hair loss can be minimized. Patients should be informed of rare, but potentially severe, acute hair loss from compromised scalp blood supply. Patients with thin scalps are at higher risk.

\section{Conclusion}

Feminization procedures have become more and more popular and it is important for plastic surgeons who are interested in treating these patients to understand the surgical approaches and how to manage patients' expectation. Spiegel reported several extremely difficult situations and challenges in treating transgender patients [14]. This can be particularly difficult when patients and doctors are not from the same cultures.

As the medical tourism trend further increases in Korea [15] and other Asian countries, familiarity with these procedures and the potential challenges in treating transgender patients are essential.

\section{Conflicts of interest}

The author has nothing to disclose. 


\section{References}

1. Ousterhout DK. Feminization of the forehead: contour changing to improve female aesthetics. Plast Reconstr Surg 1987;79:701-13.

2. Ousterhout DK, Zlotolow IM. Aesthetic improvement of the forehead utilizing methylmethacrylate onlay implants. Aesthetic Plast Surg 1990;14:281-5.

3. Ousterhout DK. Dr. Paul Tessier and facial skeletal masculinization. Ann Plast Surg 2011;67:S10-5.

4. Spiegel JH. Facial determinants of female gender and feminizing forehead cranioplasty. Laryngoscope 2011;121:250-61.

5. Sadr J, Jarudi I, Sinha P. The role of eyebrows in face recognition. Perception 2003;32:285-93.

6. Ousterhout DK. Aesthetic contouring of the bony forehead. In: Terino EO, Flowers RS, editors. The art of alloplastic facial contouring. St. Louis, MO: Mosby; 2000. p. 67-76.

7. Altman K. Facial feminization surgery: current state of the art. Int J Oral Maxillofac Surg 2012;41:885-94.
8. Capitán L, Simon D, Kaye K, Tenorio T. Facial feminization surgery: the forehead. Surgical techniques and analysis of results. Plast Reconstr Surg 2014;134:609-19.

9. Komuro Y, Nishida M, Imazawa T, Koga Y, Yanai A. Combined frontal bone reshaping and forehead lift for frontal sinus hypertrophy. Aesthetic Plast Surg 1999;23:361-3.

10. Flowers RS, Ceydeli A. The open coronal approach to forehead rejuvenation. Clin Plast Surg 2008;35:331-51.

11. Nahai FR. The varied options in brow lifting. Clin Plast Surg 2013;40:101-4.

12. Ramirez AL, Ende KH, Kabaker SS. Correction of the high female hairline. Arch Facial Plast Surg 2009;11:84-90.

13. Marten TJ. Hairline lowering during foreheadplasty. Plast Reconstr Surg 1999;103:224-36.

14. Spiegel JH. Challenges in care of the transgender patient seeking facial feminization surgery. Facial Plast Surg Clin North Am 2008;16:233-8.

15. Koh CW. Characteristics of cosmetic medical tourism in Korea. J Cosmet Med 2017;1:25-9. 